KYUNGPOOK Math. J. 54(2014), 387-399

http://dx.doi.org/10.5666/KMJ.2014.54.3.387

\title{
Weak Separation Axioms in Generalized Topological Spaces
}

\author{
V. Renukadevi \\ Department of Mathematics, ANJA College (Autonomous), Sivakasi - 626 124, \\ Tamil Nadu, India \\ e-mail : renu_siva2003@yahoo.com \\ D. SIVARAJ* \\ Department of Mathematics, G.K.M College of Engineering and Technology, New \\ Perungalathur, Chennai - 600 063, Tamil Nadu, India \\ e-mail : ttn_sivaraj@yahoo.co.in
}

ABstract. We show that in quasi-topological spaces, separation axiom $T_{2}$ is equivalent to $\alpha-T_{2}, T_{0}$ is equivalent to semi $-T_{0}$, and semi $-T_{\frac{1}{2}}$ is equivalent to semi $-T_{D}$. Also, we give characterizations for $\alpha-T_{1}$, semi $-T_{1}$ and semi $-T_{\frac{1}{2}}$ generalized topological spaces.

\section{Introduction}

A generalized topology $\mu[1]$ on a nonempty set $X$ is a collection of subsets of $X$ such that $\emptyset \in \mu$ and $\mu$ is closed under arbitrary union. Elements of $\mu$ are called $\mu$-open sets. A subset $A$ of $X$ is said to be $\mu$-closed if $X-A$ is $\mu$-open. The pair $(X, \mu)$ is called a generalized topological space (GTS). By a space $(X, \mu)$, we will always mean a $\operatorname{GTS}(X, \mu)$. For $A \subset X, c_{\mu}(A)$ is the intersection of all $\mu$-closed sets containing $A$ and $i_{\mu}(A)$ is the union of all $\mu$-open sets contained in $A$. A subset $A$ of a space $(X, \mu)$ is said to be $\mu \alpha$-open [3] (resp., $\mu$-semiopen [3], $\mu$-preopen [3], $\mu b$-open [11], $\mu \beta$-open [3] ) if $A \subset i_{\mu} c_{\mu} i_{\mu}(A)$ (resp., $A \subset$ $\left.c_{\mu} i_{\mu}(A), A \subset i_{\mu} c_{\mu}(A), A \subset i_{\mu} c_{\mu}(A) \cup c_{\mu} i_{\mu}(A), A \subset c_{\mu} i_{\mu} c_{\mu}(A)\right)$. We will denote the family of all $\mu \alpha$-open (resp., $\mu$-semiopen, $\mu$-preopen, $\mu b$-open, $\mu \beta$-open) sets by $\alpha(\mu)$ (resp., $\sigma(\mu), \pi(\mu), b(\mu), \beta(\mu)$ ) or simply, $\alpha$ (resp., $\sigma, \pi, b, \beta$ ). For $\kappa \in\{\alpha, \sigma, \pi, b, \beta\}, \kappa$ is a generalized topology such that $\alpha \subset \sigma \subset b \subset \beta, \alpha \subset \pi \subset$ $b \subset \beta$ and $\alpha=\sigma \cap \pi$. If $A$ is a subset of a space $(X, \kappa)$, then $c_{\kappa}(A)$ is the intersection of all $\kappa$-closed sets containing $A$ and $i_{\kappa}(A)$ is the union of all $\kappa$-open sets contained in $A$. Note that the operator $c_{\kappa}$ is monotonic, increasing and idempotent, and the

* Corresponding Author.

Received April 11, 2012; accepted September 17, 2012.

2010 Mathematics Subject Classification: Primary 54A05, 54D10.

Key words and phrases: generalized topology, $\mu$-closed and $\mu$-open sets, generalized $T_{i}$ spaces for $i=0, D, \frac{1}{2}, 1$ and 2 . 
operator $i_{\kappa}$ is monotonic, decreasing and idempotent on $\wp(X)$. Clearly, $A$ is $\kappa$-open if and only if $A=i_{\kappa}(A)$ and $A$ is $\kappa$-closed if and only if $A=c_{\kappa}(A)$. Also, for every subset $A$ of a space $(X, \kappa), X-i_{\kappa}(A)=c_{\kappa}(X-A)$. A subset $A$ of a space $(X, \mu)$ is said to be $\mu$-rare [9] (resp., $\mu r-$ open [2]) if $i_{\mu} c_{\mu}(A)=\emptyset$ (resp., $A=i_{\mu} c_{\mu}(A)$ ). A space $(X, \mu)$ is said to be a quasi-topological space [4], if $\mu$ is closed under finite intersection. In [4], it is established that in a quasi-topological space, $i_{\mu}$ and $c_{\mu}$ preserve finite intersection and finite union, respectively. Moreover, all the results established in [11] are true for quasi-topological spaces. A space $(X, \mu)$ is said to be strong if $X \in \mu$. If $(X, \mu)$ is a space which is not strong, then by Proposition 1.2 of [5], $X \in \sigma$ and so, it follows that $X \in b$ and $X \in \beta$. Throughout the paper, if $\mu$ is a generalized topology on $X$, let $\mathcal{M}_{\kappa}=\cup\{A \mid A \in \kappa\}$, where $\kappa \in\{\mu, \alpha, \sigma, \pi, b, \beta\}$. Since $\kappa \mid \mathcal{M}_{\kappa}=\left\{V \cap \mathcal{M}_{\kappa} \mid V \in \kappa\right\}=\kappa,\left(\mathcal{M}_{\kappa}, \kappa\right)$ is a strong subspace of $(X, \kappa)$. Moreover, always $\mathcal{M}_{\sigma}=\mathcal{M}_{b}=\mathcal{M}_{\beta}=X$ and if $\mathcal{M}_{\mu} \neq X$, then $\mathcal{M}_{\mu}=\mathcal{M}_{\alpha}=\mathcal{M}_{\pi}$.

A space $(X, \mu)$ is said to be a $T_{0}$ space [10] if for every pair of distinct points $x, y$ of $\mathcal{M}_{\mu}$, there exists a $\mu$-open set containing one of the points but not the other. $(X, \mu)$ is said to be a $T_{1}$ space [10] if for every $x \in \mathcal{M}_{\mu},\{x\} \cup\left(X-\mathcal{M}_{\mu}\right)$ is $\mu$-closed. $(X, \mu)$ is said to be a $T_{2}$ space [10] if for $x, y \in \mathcal{M}_{\mu}$ with $x \neq y$, there exist disjoint $\mu$-open sets $G$ and $H$ such that $x \in G$ and $y \in H$. A subset $A$ of $\mathcal{M}_{\mu}$ of a space $(X, \mu)$ is said to be $g_{\mu}^{\star}-$ closed [7] if $c_{\mu}(A) \cap \mathcal{M}_{\mu} \subset M$ whenever $A \subset M$ and $M \in \mu$. A subset $A$ of $\mathcal{M}_{\mu}$ in a space $(X, \mu)$ is said to be $g_{\mu}^{\star}$-open [7] if $\mathcal{M}_{\mu}-A$ is $g_{\mu}^{\star}$-closed. A space $(X, \mu)$ is said to be a $\mu-T_{\frac{1}{2}}$ space if every $g_{\mu}^{\star}$-open set is a $\mu$-open set or equivalently, every $g_{\mu}^{\star}$-closed set is a closed set in the subspace $\left(\mathcal{M}_{\mu}, \mu\right)$. The following lemmas will be useful in the sequel.

Lemma 1.1. ([9, Theorem 3.2]) Let $(X, \mu)$ be a quasi-topological space and $A$ be a $\mu$-open subset of $\mathrm{X}$. Then $c_{\mu}(A)-A$ is $\mu$-rare.

Lemma 1.2. ([9, Theorem 3.3]) Let $(X, \mu)$ be a quasi-topological space. If $A$ and $\mathrm{B}$ are $\mu$-rare subsets of $\mathrm{X}$, then $A \cup B$ is also a $\mu$-rare set.

Lemma 1.3. ([10, Theorem 3.8]) Let $(X, \mu)$ be a space. Then $(X, \mu)$ is a $T_{1}$ space if and only if $c_{\mu}(\{x\}) \cap \mathcal{M}_{\mu}=\{x\}$ for every $x \in \mathcal{M}_{\mu}$. Equivalently, $(X, \mu)$ is a $T_{1}$ space if and only if $\{x\}$ is $\mu$-closed in $\left(\mathcal{M}_{\mu}, \mu\right)$ for every $x \in \mathcal{M}_{\mu}$.

Lemma 1.4. ([7, Lemma 3.1]) Let $(X, \mu)$ be a space which is not strong and $A \subset X$. Then the following hold.

(a) $(X, \lambda)$ is a strong generalized space for every $\lambda \in\{\sigma, b, \beta\}$.

(b) $(X, \alpha)$ and $(X, \pi)$ are not strong and $\mathcal{M}_{\mu}=\mathcal{M}_{\alpha}=\mathcal{M}_{\pi}$.

(c) $X-\mathcal{M}_{\mu}$ is a $\mu$-closed set contained in every $\mu$-closed set.

(d) $A \subset \mathcal{M}_{\mu}$ is $\mu$-closed in $\left(\mathcal{M}_{\mu}, \mu\right)$ if and only if $A=c_{\mu}(A) \cap \mathcal{M}_{\mu}$.

Lemma 1.5. Let $(X, \mu)$ be a space and $A \subset X$. Then the following hold.

(a) $c_{\alpha}(A)=A \cup c_{\mu}\left(i_{\mu}\left(c_{\mu}(A)\right)\right)[3$, Lemma 2.2].

(b) $c_{\sigma}(A)=A \cup i_{\mu}\left(c_{\mu}(A)\right)[3$, Lemma 2.2]. 
(c) If $\mu$ is a quasi-topology, then $c_{\pi}(A)=A \cup c_{\mu} i_{\mu}(A), c_{b}(A)=c_{\sigma}(A) \cap c_{\pi}(A)$ and $c_{\beta}(A)=A \cup i_{\mu} c_{\mu} i_{\mu}(A)[11]$.

(d) If $\mu$ is a quasi-topology, then $B \subset \mathcal{M}_{\mu}$ is $\pi$-closed in $\left(\mathcal{M}_{\mu}, \pi\right)$ if and only if $c_{\mu} i_{\mu}(B) \cap \mathcal{M}_{\mu} \subset B$.

(e) If $\mu$ is a quasi-topology, then $B \subset X$ is $b$-closed in $X$ if and only if $i_{\mu} c_{\mu}(B) \cap c_{\mu} i_{\mu}(B) \subset B$.

(f) If $\mu$ is a quasi-topology, then $B \subset X$ is $\beta$-closed in $X$ if and only if $i_{\mu} c_{\mu} i_{\mu}(B) \subset B$.

(g) $B \subset \mathcal{M}_{\mu}$ is $\alpha$-closed in $\left(\mathcal{M}_{\mu}, \alpha\right)$ if and only if $c_{\mu} i_{\mu} c_{\mu}(B) \cap \mathcal{M}_{\mu} \subset B$.

Proof. (d) By Lemma 1.4(d), $B \subset \mathcal{M}_{\mu}$ is $\pi$-closed in $\left(\mathcal{M}_{\mu}, \pi\right)$ if and only if $A=$ $c_{\pi}(A) \cap \mathcal{M}_{\mu}$ if and only if $B=\left(B \cup c_{\mu} i_{\mu}(B)\right) \cap \mathcal{M}_{\mu}=B \cup\left(c_{\mu} i_{\mu}(B) \cap \mathcal{M}_{\mu}\right)$. Therefore, $c_{\mu} i_{\mu}(B) \cap \mathcal{M}_{\mu} \subset B$. The proof of (e) and (f) are similar to the proof of (d) in which $\mathcal{M}_{b}=\mathcal{M}_{\beta}=X$ and the proof of $(\mathrm{g})$ is similar to the proof of $(\mathrm{d})$.

Lemma 1.6.([10, Theorem 3.4]) Let $(X, \mu)$ be a space which is not strong. Then $(X, \mu)$ is $T_{0}$ if and only if for every pair of distinct points $x$ and $y$ in $\mathcal{M}_{\mu}, c_{\mu}(\{x\}) \neq$ $c_{\mu}(\{y\})$.

Lemma 1.7.([10, Theorem 3.24]) A space $(X, \mu)$ is $\mu-T_{\frac{1}{2}}$ if and only if each $x \in \mathcal{M}_{\mu}$ is either $\mu$-open or $\mu$-closed in $\left(\mathcal{M}_{\mu}, \mu\right)$.

Theorem 1.8. Let $(X, \tau)$ be a space and $x \in X$. Then the following hold.

(a) Every subset $A$ of $X-\mathcal{M}_{\mu}$ is $\mu$-rare and $\{x\} \subset \mathcal{M}_{\mu}$ is either $\pi$-open or $\mu$-rare.

(b) For every $x \in \mathcal{M}_{\mu},\{x\}$ is $\pi$-open if and only if $\{x\}$ is $\beta$-open.

(c) For every $x \in \mathcal{M}_{\mu},\{x\}$ is $\mu$-open if and only if $\{x\}$ is $\alpha$-open.

(d) For every $x \in \mathcal{M}_{\mu},\{x\}$ is $\mu$-open if and only if $\{x\}$ is $\alpha$-open if and only if $\{x\}$ is $\sigma$-open.

(e) For every $x \in \mathcal{M}_{\mu}$, if $\{x\}$ is $\mu$-rare, then $\{x\}$ is $\alpha$-closed in $\left(\mathcal{M}_{\mu}, \alpha\right)$. Hence $\{x\}$ is $\sigma$-closed, $b$-closed and $\beta$-closed.

(f) For every $x \in \mathcal{M}_{\mu},\{x\}$ is $\sigma$-closed if and only if $\{x\}$ is $\mu$-rare or $\mu r$-open.

Proof. (a) If $A \subset X-\mathcal{M}_{\mu}$, then $c_{\mu}(A)=X-\mathcal{M}_{\mu}$ so that $i_{\mu} c_{\mu}(A)=\emptyset$ and so $A$ is $\mu$-rare. Suppose $x \in \mathcal{M}_{\mu}$. If $i_{\mu} c_{\mu}(\{x\})=\emptyset$, then $\{x\}$ is $\mu$-rare. Suppose that $i_{\mu} c_{\mu}(\{x\}) \neq \emptyset$. Then $y \in i_{\mu} c_{\mu}(\{x\})$. Since $y \in c_{\mu}(\{x\})$ and $i_{\mu} c_{\mu}(\{x\})$ is a $\mu$-open set containing $y, i_{\mu} c_{\mu}(\{x\}) \cap\{x\} \neq \emptyset$ and so $x \in i_{\mu} c_{\mu}(\{x\})$. Hence $\{x\}$ is $\pi$-open. (b) Suppose that $x \in \mathcal{M}_{\mu}$ and $\{x\}$ is $\beta$-open. Then $\{x\} \subset c_{\mu} i_{\mu} c_{\mu}(\{x\})$. If $i_{\mu} c_{\mu}(\{x\})=\emptyset$, then $c_{\mu} i_{\mu} c_{\mu}(\{x\})=X-\mathcal{M}_{\mu}$ and so $x \in X-\mathcal{M}_{\mu}$, a contradiction. Hence $i_{\mu} c_{\mu}(\{x\}) \neq \emptyset$. As in the proof of (a), we can prove that $\{x\}$ is $\pi$-open. The converse is clear.

(c) Let $x \in X$ and $\{x\}$ be $\alpha$-open. Then $\{x\} \subset i_{\mu} c_{\mu} i_{\mu}(\{x\})$. Suppose $i_{\mu}(\{x\})=\emptyset$. Then $\{x\} \subset i_{\mu} c_{\mu} i_{\mu}(\{x\})=i_{\mu} c_{\mu}(\emptyset)=i_{\mu}\left(X-\mathcal{M}_{\mu}\right)=\emptyset$, a contradiction and so 
$i_{\mu}(\{x\}) \neq \emptyset$, which implies that $i_{\mu}(\{x\})=\{x\}$. The converse is clear.

(d) Suppose $x \in \mathcal{M}_{\mu}$ and $\{x\}$ is $\sigma$-open. Then $\{x\} \subset c_{\mu} i_{\mu}(\{x\})$ which implies that $i_{\mu}(\{x\}) \neq \emptyset$ and so $i_{\mu}(\{x\})=\{x\}$. Hence $\{x\}$ is $\mu$-open.

(e) Let $x \in \mathcal{M}_{\mu}$ and $\{x\}$ be $\mu$-rare. Then $i_{\mu} c_{\mu}(\{x\})=\emptyset$. Now $c_{\alpha}(\{x\}) \cap \mathcal{M}_{\mu}=$ $\left(c_{\mu} i_{\mu} c_{\mu}(\{x\}) \cup\{x\}\right) \cap \mathcal{M}_{\mu}=\left(c_{\mu}(\emptyset) \cup\{x\}\right) \cap \mathcal{M}_{\mu}=\left(\left(X-\mathcal{M}_{\mu}\right) \cup\{x\}\right) \cap \mathcal{M}_{\mu}=\{x\}$ and so $\{x\}$ is $\alpha$-closed in $\left(\mathcal{M}_{\mu}, \alpha\right)$.

(f) Proof is clear.

In every topological space $(X, \tau)$, for each point $x \in X$, either $\{x\}$ is open or $\{x\}$ is preclosed. The following Example 1.9 shows that the statement for $x \in \mathcal{M}_{\mu},\{x\}$ is either $\mu$-open or $\pi$-closed need not be true in a quasi-topological space.

Example 1.9. Consider the space $(X, \mu)$ where $X=\{a, b, c, d\}$ and $\mu=$ $\{\emptyset,\{a\},\{b, c\},\{a, b, c\}\}$. Clearly, the space is a quasi-topological space. For $b \in$ $\mathcal{M}_{\mu}=\{a, b, c\}, c_{\mu} i_{\mu}(\{b\})=c_{\mu}(\emptyset)=\{d\} \not \subset\{b\}$ and so $\{b\}$ is not $\pi$-closed. Note that $\{b\}$ is not $\mu$-open.

\section{Main Results}

Let $(X, \mu)$ be a space and $\kappa \in\{\alpha, \sigma, \pi, b, \beta\}$. Then the space $(X, \mu)$ is said to be $\kappa-T_{i}[10]$ if and only if $(X, \kappa)$ is $T_{i}$ for $i=0, \frac{1}{2}, 1,2$. By Lemma 1.4(b), we have for $i=0,1,2, T_{i} \Rightarrow \alpha-T_{i} \Rightarrow \pi-T_{i}$ and by Lemma 1.4(a), we have for $i=0,1,2, \sigma-T_{i} \Rightarrow b-T_{i} \Rightarrow \beta-T_{i}$. The following Theorem 2.1 gives a characterization of $\mu \alpha$-open sets of quasi-topological spaces. Example 2.2 below shows that the condition quasi-topology on the space $(X, \mu)$ cannot be dropped in Theorem 2.1.

Theorem 2.1. Let $(X, \mu)$ be a quasi-topological space. Then $\alpha=\{U-R \mid U \in \mu$ and $R$ is $\mu$-rare $\}$.

Proof. Suppose $A \in \alpha$. Then $A \subset i_{\mu} c_{\mu} i_{\mu}(A)$ and $A=i_{\mu} c_{\mu} i_{\mu}(A)-\left(i_{\mu} c_{\mu} i_{\mu}(A)-A\right)=$ $U-R$. Now $i_{\mu} c_{\mu}(R)=i_{\mu} c_{\mu}\left(i_{\mu} c_{\mu} i_{\mu}(A)-A\right) \subset i_{\mu}\left(c_{\mu} i_{\mu}(A)-i_{\mu}(A)\right)=\emptyset$, by Lemma 1.1 and so $R=i_{\mu} c_{\mu} i_{\mu}(A)-A$ is a $\mu$-rare set. Conversely, suppose that $A=U-R$ where $U \in \mu$ and $R$ is $\mu$-rare. Then $c_{\mu} i_{\mu}(A)=c_{\mu} i_{\mu}(U-R)=c_{\mu}\left(i_{\mu}(U)-c_{\mu}(R)\right) \supset$ $i_{\mu}(U)-i_{\mu} c_{\mu}(R)=i_{\mu}(U)=U$. Thus, $U \subset c_{\mu} i_{\mu}(A)$ which implies that $U \subset i_{\mu} c_{\mu} i_{\mu}(A)$. Since $A \subset U, A \subset i_{\mu} c_{\mu} i_{\mu}(A)$ and so $A \in \alpha$.

Example 2.2. Consider the space $(X, \mu)$ where $X=\{a, b, c, d\}$ and $\mu=$ $\{\emptyset,\{a\},\{a, b\},\{b, c\},\{a, b, c\}\}$. Note that $\mu$ is not a quasi-topological space and $\alpha=\{\emptyset,\{a\},\{a, b\},\{b, c\},\{a, b, c\}\}$. If $A=\{b\}$, then $A=\{b, c\}-\{c\}$, where $\{c\}$ is $\mu$-rare. But $A \notin \alpha$.

In Theorem 2 of [8], it is established that a topological space $(X, \tau)$ is $T_{2}$ if and only if $\left(X, \tau^{\alpha}\right)$ is $T_{2}$ where $\tau^{\alpha}$ is the family of all $\alpha$-open sets of the topological space $(X, \tau)$. The following Theorem 2.3 is a generalization of the above result. Theorem 2.4 below shows that every $\alpha-T_{2}$ space is a $\sigma-T_{2}$ space. 
Theorem 2.3. Let $(X, \mu)$ be a quasi-topological space. Then $(X, \mu)$ is $T_{2}$ if and only if $(X, \alpha)$ is $T_{2}$.

Proof. Suppose that $(X, \alpha)$ is $T_{2}$. Let $x, y \in \mathcal{M}_{\mu}$ with $x \neq y$. Then there exist disjoint $\alpha$-open sets $U$ and $V$ such that $x \in U$ and $y \in V$. By Theorem 2.1, there exist $\mu$-open sets $G, H$ and $\mu$-rare sets $I, J$ such that $U=G-I$ and $V=H-J$. Now $U \cap V=\emptyset$ implies that $(G \cap H)-(I \cup J)=\emptyset$ and so $G \cap H \subset I \cup J \subset c_{\mu}(I \cup J)$. Therefore, $i_{\mu}(G \cap H) \subset i_{\mu} c_{\mu}(I \cup J)$ which implies that $G \cap H \subset i_{\mu} c_{\mu}(I \cup J)$. Since $I$ and $J$ are $\mu$-rare sets, by Lemma $1.2, I \cup J$ is a $\mu$-rare set and so $G \cap H=\emptyset$. Hence $(X, \mu)$ is $T_{2}$, since $x \in G$ and $y \in H$. The converse is clear.

Theorem 2.4. Every $\alpha-T_{2}$ space $(X, \mu)$ is a $\sigma-T_{2}$ space as well as a $\pi-T_{2}$ space.

Proof. Suppose that $(X, \mu)$ is an $\alpha-T_{2}$ space. Let $x, y \in X$ such that $x \neq y$. If $x, y \in \mathcal{M}_{\mu}$, then the points are separated by disjoint $\alpha$-open sets and hence are separated by disjoint $\sigma$-open sets. If $x \in \mathcal{M}_{\mu}$ and $y \in X-\mathcal{M}_{\mu}$, then $\mathcal{M}_{\mu}$ and $\{y\}$ are the disjoint $\sigma$-open sets containing $x$ and $y$, respectively, since $c_{\mu} i_{\mu}(\{y\})=$ $c_{\mu}(\emptyset)=X-\mathcal{M}_{\mu}$. If $x, y \in X-\mathcal{M}_{\mu}$, then $\{x\}$ and $\{y\}$ are the disjoint $\sigma$-open sets containing $x$ and $y$, respectively. Therefore, $(X, \mu)$ is a $\sigma-T_{2}$ space. Clearly, every $\alpha-T_{2}$ space $(X, \mu)$ is a $\pi-T_{2}$ space.

Theorem 2.5. Every $\pi-T_{2}$ space $(X, \mu)$ is a $b-T_{2}$ space.

Proof. Let $x, y \in X$ such that $x \neq y$. If $x, y \in \mathcal{M}_{\mu}$, then the proof is clear. Suppose that $x \in \mathcal{M}_{\mu}$ and $y \notin \mathcal{M}_{\mu}$. Then $c_{\mu} i_{\mu}(\{y\}) \cup i_{\mu} c_{\mu}(\{y\})=c_{\mu}(\emptyset) \cup i_{\mu}\left(X-\mathcal{M}_{\mu}\right)=$ $X-\mathcal{M}_{\mu} \supset\{y\}$ which implies that $\{y\} \in b$. So $\mathcal{M}_{\mu}$ and $\{y\}$ are the required disjoint $b$-open sets containing $x$ and $y$. If $x \notin \mathcal{M}_{\mu}$ and $y \notin \mathcal{M}_{\mu}$, then $\{x\}$ and $\{y\}$ are the required disjoint $b$-open sets containing $x$ and $y$. Therefore, $(X, \mu)$ is $b-T_{2}$.

Remark 2.6. (a) In every space $(X, \mu)$, since every $\alpha$-open (resp., $\pi$-open) set is a subset of some $\mu$-open set, we cannot find $\alpha$-open (resp., $\pi$-open) sets containing points of $X-\mathcal{M}_{\mu}$.

(b) One can easily verify that the converse part of Theorems 2.4 and 2.5 are not true. Moreover, it is well known that they are not true even in topological spaces. The following Theorem 2.7 gives a characterization of $\alpha-T_{1}$ spaces and Theorem 2.8 below gives a characterization of $\alpha-T_{0}$ spaces.

Theorem 2.7. A quasi-topological space $(X, \mu)$ is $\alpha-T_{1}$ if and only if $(X, \mu)$ is both $\sigma-T_{1}$ and $\pi-T_{1}$.

Proof. Clearly, $(X, \mu)$ is $\alpha-T_{1}$ implies that $(X, \mu)$ is $\pi-T_{1}$. Let $x \in X$. If $x \in \mathcal{M}_{\mu}$, then $\{x\}$ is $\sigma$-closed, by hypothesis. If $x \notin \mathcal{M}_{\mu}$, then $c_{\sigma}(\{x\})=i_{\mu} c_{\mu}(\{x\}) \cup$ $\{x\}=i_{\mu}\left(X-\mathcal{M}_{\mu}\right) \cup\{x\}=\{x\}$ and so $\{x\}$ is $\sigma$-closed. Hence $(X, \mu)$ is $\sigma-T_{1}$. Suppose $(X, \mu)$ is both $\sigma-T_{1}$ and $\pi-T_{1}$. Let $x \in \mathcal{M}_{\mu}$. Now, $(X, \mu)$ is $\pi-T_{1}$ 
implies that $\{x\}$ is $\pi$-closed and $(X, \mu)$ is $\sigma-T_{1}$ implies that $c_{\sigma}(\{x\})=\{x\}$. Since $i_{\mu} c_{\mu}(\{x\}) \cap \mathcal{M}_{\mu} \subset\{x\}$, by Lemma 1.5(d), we have $i_{\mu} c_{\mu}\left(c_{\sigma}(\{x\})\right) \cap \mathcal{M}_{\mu} \subset\{x\}$ which in turn implies that $c_{\mu} i_{\mu} c_{\mu}(\{x\}) \cap \mathcal{M}_{\mu} \subset\{x\}$, by Lemma 2.4 of [11]. By Lemma $1.5(\mathrm{~g}),(X, \mu)$ is $\alpha-T_{1}$.

Theorem 2.8. If $(X, \mu)$ is a quasi-topological space, then $(X, \alpha)$ is $T_{0}$ if and only if $(X, \sigma)$ is $T_{0}$.

Proof. Suppose $(X, \sigma)$ is $T_{0}$. If $(X, \alpha)$ is not $T_{0}$, then by Lemma 1.6, there exist distinct points $x$ and $y \in \mathcal{M}_{\mu}$ such that $c_{\alpha}(\{x\})=c_{\alpha}(\{y\})$ which implies that $\{x\} \cup c_{\mu} i_{\mu} c_{\mu}(\{x\})=\{y\} \cup c_{\mu} i_{\mu} c_{\mu}(\{y\})$, by Lemma 1.5(a) and so $x \in c_{\mu} i_{\mu} c_{\mu}(\{y\})$ and $y \in c_{\mu} i_{\mu} c_{\mu}(\{x\})$, since $x \neq y$. Since $(X, \sigma)$ is $T_{0}$, either $x \notin c_{\sigma}(\{y\})$ or $y \notin$ $c_{\sigma}(\{x\})$. Suppose that $x \notin c_{\sigma}(\{y\})=\{y\} \cup i_{\mu} c_{\mu}(\{y\})$, by Lemma 1.5(b). Now $c_{\mu} i_{\mu} c_{\mu}(\{x\}) \neq \emptyset$ implies that $i_{\mu} c_{\mu}(\{x\}) \neq \emptyset$, since $y \in \mathcal{M}_{\mu}$. Let $z \in i_{\mu} c_{\mu}(\{x\})$. Since $i_{\mu} c_{\mu}(\{x\})$ is $\mu$-open and $z \in c_{\mu}(\{x\})$, it follows that $x \in i_{\mu} c_{\mu}(\{x\})$ and so $i_{\mu} c_{\mu}(\{x\})$ is a $\mu$-open set containing $x$. Since $x \in c_{\mu} i_{\mu} c_{\mu}(\{y\}), i_{\mu} c_{\mu}(\{y\}) \cap i_{\mu} c_{\mu}(\{x\}) \neq \emptyset$ and so $i_{\mu}\left(c_{\mu}(\{y\}) \cap c_{\mu}(\{x\})\right) \neq \emptyset$, since $(X, \mu)$ is a quasi-topological space. Let $u \in i_{\mu}\left(c_{\mu}(\{y\}) \cap c_{\mu}(\{x\})\right)$. Then $i_{\mu}\left(c_{\mu}(\{y\}) \cap c_{\mu}(\{x\})\right)$ is a $\mu$-open set containing $u$ and also $u \in c_{\mu}(\{y\}) \cap c_{\mu}(\{x\})$. Hence $x, y \in i_{\mu} c_{\mu}(\{y\}) \cap i_{\mu} c_{\mu}(\{x\})$ and so $x \in$ $\{y\} \cup i_{\mu} c_{\mu}(\{y\})=c_{\sigma}(\{y\})$, a contradiction. Therefore, $(X, \mu)$ is $\alpha-T_{0}$. Conversely, suppose that $(X, \mu)$ is $\alpha-T_{0}$. Let $x, y \in X=\mathcal{M}_{\sigma}$ with $x \neq y$. If $x, y \in \mathcal{M}_{\mu}$, then there exists $U \in \alpha$ containing one point but not the other point. Since $\alpha \subset \sigma,(X, \mu)$ is $\sigma-T_{0}$. Suppose $x \in \mathcal{M}_{\mu}$ and $y \notin \mathcal{M}_{\mu}$. If $X-\mathcal{M}_{\mu}=\{y\}$, then $\{y\}$ is $\mu$-closed and hence $\sigma$-closed. Thus, $x \notin c_{\sigma}(\{y\})$ which implies that $c_{\sigma}(\{x\}) \neq c_{\sigma}(\{y\})$. If $X-\mathcal{M}_{\mu}$ contains points other than $y$, then $c_{\mu}(\{y\})=X-\mathcal{M}_{\mu}$ and $x \notin X-\mathcal{M}_{\mu}$ which implies that $c_{\sigma}(\{y\}) \subset X-\mathcal{M}_{\mu}$ and so $c_{\sigma}(\{x\}) \neq c_{\sigma}(\{y\})$, since $x \neq y$. If $x \notin \mathcal{M}_{\mu}$ and $y \notin \mathcal{M}_{\mu}$, then $c_{\mu}(\{x\})=X-\mathcal{M}_{\mu}=c_{\mu}(\{y\})$. By Lemma 1.5(b), $c_{\sigma}(\{x\})=\{x\} \cup i_{\mu} c_{\mu}(\{x\})=\{x\} \cup i_{\mu}\left(X-\mathcal{M}_{\mu}\right)=\{x\} \cup \emptyset=\{x\}$. Similarly, $c_{\sigma}(\{y\})=\{y\}$. Since $x \neq y, c_{\sigma}(\{x\}) \neq c_{\sigma}(\{y\})$. Hence by Lemma 1.6, $(X, \mu)$ is $\sigma-T_{0}$.

The following Theorem 2.9 gives a characterization of $\alpha-T_{1}$ spaces and Theorem 2.10 below gives a characterization of $\sigma-T_{1}$ spaces.

Theorem 2.9. A space $(X, \mu)$ is $\alpha-T_{1}$ if and only if for every $x \in \mathcal{M}_{\mu}$, either $\{x\}$ is $\mu$-rare or $c_{\mu}(\{x\}) \cap \mathcal{M}_{\mu}=\{x\}$.

Proof. Suppose that $(X, \mu)$ is $\alpha-T_{1}$. Let $x \in \mathcal{M}_{\mu}$. Then by Lemma 1.3, $c_{\alpha}(\{x\}) \cap \mathcal{M}_{\alpha}=c_{\alpha}(\{x\}) \cap \mathcal{M}_{\mu}=\{x\}$, since $\mathcal{M}_{\mu}=\mathcal{M}_{\alpha}$. Therefore, by Lemma 1.5(a), $\left(\{x\} \cup c_{\mu} i_{\mu} c_{\mu}(\{x\})\right) \cap \mathcal{M}_{\mu}=\{x\}$ and so $\{x\} \cup\left(c_{\mu} i_{\mu} c_{\mu}(\{x\}) \cap \mathcal{M}_{\mu}\right)=\{x\}$ which implies that either $c_{\mu} i_{\mu} c_{\mu}(\{x\}) \cap \mathcal{M}_{\mu}=\emptyset$ or $c_{\mu} i_{\mu} c_{\mu}(\{x\}) \cap \mathcal{M}_{\mu}=\{x\}$. If $c_{\mu} i_{\mu} c_{\mu}(\{x\}) \cap \mathcal{M}_{\mu}=\emptyset$, then $c_{\mu} i_{\mu} c_{\mu}(\{x\})=X-\mathcal{M}_{\mu}$ which implies that $i_{\mu} c_{\mu} i_{\mu} c_{\mu}(\{x\})=i_{\mu}\left(X-\mathcal{M}_{\mu}\right)=\emptyset$ which in turn implies that $i_{\mu} c_{\mu}(\{x\})=\emptyset$ and so $\{x\}$ is $\mu$-rare. If $c_{\mu} i_{\mu} c_{\mu}(\{x\}) \cap \mathcal{M}_{\mu}=\{x\}$, then $i_{\mu} c_{\mu}(\{x\}) \neq \emptyset$. For, if $i_{\mu} c_{\mu}(\{x\})=\emptyset$, then $c_{\mu} i_{\mu} c_{\mu}(\{x\}) \cap \mathcal{M}_{\mu}=c_{\mu}(\emptyset) \cap \mathcal{M}_{\mu}=\left(X-\mathcal{M}_{\mu}\right) \cap \mathcal{M}_{\mu}=\emptyset$, 
a contradiction. Since $\emptyset \neq i_{\mu} c_{\mu}(\{x\}) \subset c_{\mu} i_{\mu} c_{\mu}(\{x\}) \cap \mathcal{M}_{\mu}=\{x\}$, it follows that $i_{\mu} c_{\mu}(\{x\})=\{x\}$ which implies that $c_{\mu}(\{x\}) \cap \mathcal{M}_{\mu}=\{x\}$. Conversely, let $x \in \mathcal{M}_{\alpha}$. Since $\mathcal{M}_{\alpha}=\mathcal{M}_{\mu}, x \in \mathcal{M}_{\mu}$. If $\{x\}$ is $\mu$-rare, then $i_{\mu} c_{\mu}(\{x\})=\emptyset$. Therefore, $c_{\alpha}(\{x\}) \cap \mathcal{M}_{\alpha}=\left(\{x\} \cup c_{\mu} i_{\mu} c_{\mu}(\{x\})\right) \cap \mathcal{M}_{\alpha}=\left(\{x\} \cup c_{\mu}(\emptyset)\right) \cap \mathcal{M}_{\alpha}=$ $\left(\{x\} \cup\left(X-\mathcal{M}_{\mu}\right)\right) \cap \mathcal{M}_{\mu}=\{x\} \cap \mathcal{M}_{\mu}=\{x\}$. On the other hand, if $c_{\mu}(\{x\}) \cap \mathcal{M}_{\mu}=\{x\}$, then $\{x\} \subset c_{\alpha}(\{x\}) \cap \mathcal{M}_{\alpha} \subset c_{\mu}(\{x\}) \cap \mathcal{M}_{\mu}=\{x\}$ and so $c_{\alpha}(\{x\}) \cap \mathcal{M}_{\alpha}=\{x\}$. Hence by Lemma $1.3,(X, \mu)$ is $\alpha-T_{1}$.

Theorem 2.10. A space $(X, \mu)$ is $\sigma-T_{1}$ if and only if for every $x \in X$, either $\{x\}$ is $\mu$-rare or $\{x\}$ is $\mu r-$ open.

Proof. Let $x \in X$. If $\{x\}$ is $\mu$-rare, then $i_{\mu} c_{\mu}(\{x\})=\emptyset$ and so $c_{\sigma}(\{x\})=\{x\} \cup$ $i_{\mu} c_{\mu}(\{x\})=\{x\}$. If $\{x\}$ is $\mu r$-open, then $i_{\mu} c_{\mu}(\{x\})=\{x\}$ which implies that $c_{\sigma}(\{x\})=\{x\}$. Therefore, by Lemma $1.3,(X, \mu)$ is $\sigma-T_{1}$. Conversely, let $x \in X$. Since $(X, \mu)$ is $\sigma-T_{1}$, by Lemma $1.3,\{x\}=c_{\sigma}(\{x\})$ which implies that $\{x\}=$ $\{x\} \cup i_{\mu} c_{\mu}(\{x\})$ and so $i_{\mu} c_{\mu}(\{x\}) \subset\{x\}$. If $i_{\mu} c_{\mu}(\{x\})=\emptyset$, then $\{x\}$ is $\mu$-rare. If $i_{\mu} c_{\mu}(\{x\})=\{x\}$, then $\{x\}$ is $\mu r-$ open.

Corollary 2.11. Every $\alpha-T_{1}$ space $(X, \mu)$ is a $\sigma-T_{1}$ space.

Corollary 2.12. If $(X, \mu)$ is a space and $\kappa \in\{\alpha, \sigma, \pi, b, \beta\}$, then $(X, \mu)$ is a $\kappa-T_{\frac{1}{2}}$ space if and only if each $x \in \mathcal{M}_{\kappa}$ is either $\kappa$-open or $\kappa$-closed in $\left(\mathcal{M}_{\kappa}, \kappa\right)$.

Corollary 2.13. For $\kappa \in\{\mu, \alpha, \sigma, \pi, b, \beta\}$, every $\kappa-T_{1}$ space $(X, \mu)$ is a $\kappa-T_{\frac{1}{2}}$ space.

Proof. The proof follows from Lemma 1.7 and Lemma 1,3.

The following Theorem 2.14 gives a characterization of $\sigma-T_{\frac{1}{2}}$ spaces.

Theorem 2.14. A space $(X, \mu)$ is a $\sigma-T_{\frac{1}{2}}$ space if and only if for every $x \in X,\{x\}$ is either $\mu$-rare or $\mu$-open.

Proof. Suppose that for every $x \in X,\{x\}$ is either $\mu$-rare or $\mu$-open. If $\{x\}$ is $\mu$-rare, then $i_{\mu} c_{\mu}(\{x\})=\emptyset \subset\{x\}$ and so $\{x\}$ is $\sigma$-closed. Therefore, for each $x \in$ $X$, either $\{x\}$ is $\sigma$-closed or $\sigma$-open. Hence $(X, \mu)$ is $\sigma-T_{\frac{1}{2}}$. Conversely, let $x \in X$. Then $\{x\}$ is either $\sigma$-open or $\sigma$-closed. If $\{x\}$ is $\sigma$-open, then $\{x\} \subset c_{\mu} i_{\mu}(\{x\})$ which implies that $i_{\mu} c_{\mu}(\{x\}) \subset i_{\mu} c_{\mu} i_{\mu}(\{x\})$. If $i_{\mu}(\{x\})=\emptyset$, then $i_{\mu} c_{\mu} i_{\mu}(\{x\})=\emptyset$ and so $i_{\mu} c_{\mu}(\{x\})=\emptyset$. Hence $\{x\}$ is $\mu$-rare. If $i_{\mu}(\{x\}) \neq \emptyset$, then $\{x\}$ is $\mu$-open. Suppose that $\{x\}$ is $\sigma$-closed. Then by Lemma 1.7(f), $\{x\}$ is either $\mu$-open or $\mu$-rare. This completes the proof.

Let $(X, \mu)$ be a space and $\kappa \in\{\mu, \alpha, \sigma, \pi, b, \beta\}$. A subset $A$ of $\mathcal{M}_{\kappa}$ in a space $(X, \mu)$ is said to be $\kappa$-locally closed if $A=U \cap V$ where $U$ is $\kappa$-open and $V$ is $\kappa$-closed in $\left(\mathcal{M}_{\kappa}, \kappa\right)$. A space $(X, \mu)$ is said to be a $\kappa-T_{D}$ space if $\{x\}$ is $\kappa$-locally 
closed for every $x \in \mathcal{M}_{\kappa}$. Note that, if $(X, \mu)$ is not strong, then for $\lambda \in\{\sigma, b, \beta\}$, every $\lambda$-open as well as $\lambda$-closed sets are $\lambda$-locally closed sets. The following Lemma 2.15, gives some expected relations between $\kappa-T_{\frac{1}{2}}$ and $\kappa-T_{D}$ spaces. In Theorem 2.16 below, we show that in quasi-topological spaces, the concepts $\sigma-T_{\frac{1}{2}}$ and $\sigma-T_{D}$ are identical. Example 2.17 below shows that the condition quasitopology on $\mu$ cannot be dropped in Theorem 2.16.

Lemma 2.15. Let $(X, \mu)$ be a space. Then the following hold.

(a) For $\lambda \in\{\sigma, b, \beta\}$, if $(X, \mu)$ is $\lambda-T_{\frac{1}{2}}$, then $(X, \mu)$ is $\lambda-T_{D}$.

(b) For $\lambda \in\{\mu, \alpha, \pi\}$, if $(X, \mu)$ is $\lambda-T_{\frac{1}{2}}$, then $(X, \mu)$ is $\lambda-T_{D}$.

(c) If $(X, \mu)$ is $\alpha-T_{D}$, then $(X, \mu)$ is $\sigma-T_{D}$.

Proof. (a) Suppose that $(X, \mu)$ is a $\lambda-T_{\frac{1}{2}}$ space. Let $x \in X=\mathcal{M}_{\lambda}$. Then by Lemma 1.7, $\{x\}$ is either $\lambda$-open or $\lambda$-closed in $\left(\mathcal{M}_{\lambda}, \lambda\right)$ and so $\{x\}$ is $\lambda$-locally closed. Hence $(X, \mu)$ is $\lambda-T_{D}$.

(b) Let $x \in \mathcal{M}_{\lambda}$. Then by Lemma $1.7,\{x\}$ is either $\lambda$-open or $\lambda$-closed in $\left(\mathcal{M}_{\lambda}, \lambda\right)$. If $\{x\}$ is $\lambda$-closed in $\left(\mathcal{M}_{\lambda}, \lambda\right)$, then $\{x\}=\{x\} \cup\left(X-\mathcal{M}_{\lambda}\right)=F$ and so $\{x\}=F \cap \mathcal{M}_{\lambda}$. Hence $\{x\}$ is the intersection of a $\lambda$-open set $\mathcal{M}_{\lambda}$ and a $\lambda$-closed set $F$. If $\{x\}$ is open in $\left(\mathcal{M}_{\lambda}, \lambda\right)$, then $\{x\}=\{x\} \cap \mathcal{M}_{\lambda}$, an intersection of a $\lambda$-open set $\{x\}$ and a $\lambda$-closed set $\mathcal{M}_{\lambda}$ in $\left(M_{\lambda}, \lambda\right)$. Hence for every point $x$ in $\mathcal{M}_{\lambda},\{x\}$ is $\lambda$-locally closed. This completes the proof.

(c) Suppose $(X, \mu)$ is $\alpha-T_{D}$. Let $x \in \mathcal{M}_{\sigma}=X$. If $x \in X-\mathcal{M}_{\mu}$, then $\{x\}$ is $\sigma$-open. Then $\{x\}=\{x\} \cap X$, an intersection of a $\sigma$-open and a $\sigma$-closed set. If $x \in \mathcal{M}_{\mu}$, then $\{x\}=U \cap V$ where $U$ is $\alpha$-open and $V$ is $\alpha$-closed. Since $\alpha \subset \sigma,\{x\}$ is an intersection of a $\sigma$-open set and a $\sigma$-closed set. Hence $(X, \mu)$ is $\sigma-T_{D}$.

Theorem 2.16. Let $(X, \mu)$ be a quasi-topological space. Then $(X, \mu)$ is a $\sigma-T_{\frac{1}{2}}$ space if and only if $(X, \mu)$ is a $\sigma-T_{D}$ space.

Proof. Suppose that $(X, \mu)$ is a $\sigma-T_{\frac{1}{2}}$ space. By Lemma 2.15(a), $(X, \mu)$ is a $\sigma-T_{D}$ space. Conversely, assume that $X$ is $\sigma-T_{D}$ and $x \in X$. If $\{x\}$ is not $\mu$-rare, then $i_{\mu} c_{\mu}(\{x\}) \neq \emptyset$ and so $x \in i_{\mu} c_{\mu}(\{x\})$. Since $(X, \mu)$ is $\sigma-T_{D},\{x\}$ is $\sigma$-locally closed which implies that $c_{\sigma}(\{x\})-\{x\}$ is $\sigma$ - closed, by Theorem 2.8 of [9]. This implies that $\left(\{x\} \cup i_{\mu} c_{\mu}(\{x\})\right)-\{x\}$ is $\sigma$-closed and so $i_{\mu} c_{\mu}(\{x\})-\{x\}$ is $\sigma$-closed. Hence $\{x\} \cup\left(X-i_{\mu} c_{\mu}(\{x\})\right)$ is $\sigma$-open. Therefore, there exists $G \in \mu$ such that $G \subset\{x\} \cup\left(X-i_{\mu} c_{\mu}(\{x\})\right) \subset c_{\mu}(G)$. Since $x \in c_{\mu}(G), G \cap i_{\mu} c_{\mu}(\{x\}) \neq \emptyset$. Suppose $x \notin G$. If $\left(i_{\mu} c_{\mu}(\{x\})-\{x\}\right) \cap G=\emptyset$, then $i_{\mu} c_{\mu}(\{x\}) \cap G=\{x\}$ and so $x \in G$, which is a contradiction. Hence $\left(i_{\mu} c_{\mu}(\{x\})-\{x\}\right) \cap G \neq \emptyset$ which implies that $\left(i_{\mu} c_{\mu}(\{x\})-\{x\}\right) \cap\left(\{x\} \cup\left(X-i_{\mu} c_{\mu}(\{x\})\right)\right) \neq \emptyset$, which is not possible. Therefore, $x \in G$. Hence $i_{\mu} c_{\mu}(\{x\}) \cap G=\{x\}$ is $\mu$-open in $X$.

Example 2.17. Consider the space $(X, \mu)$ of Example 2.2. Clearly, $(X, \mu)$ is $\sigma-T_{D}$. Since $\{b\}$ is neither $\sigma$-open nor $\sigma$-closed, by Lemma 1.7, $(X, \mu)$ is not a $\sigma-T_{\frac{1}{2}}$ 
space. Note that, the space is not a quasi-topological space.

The following Theorem 2.18 shows that the separation axioms $\alpha-T_{\frac{1}{2}}$ and $\sigma-T_{\frac{1}{2}}$ are equivalent.

Theorem 2.18. Let $(X, \mu)$ be a space. Then $(X, \mu)$ is $\alpha-T_{\frac{1}{2}}$ if and only if $(X, \mu)$ is $\sigma-T_{\frac{1}{2}}$.

Proof. Let $(X, \mu)$ be a $\sigma-T_{\frac{1}{2}}$ space and $x \in \mathcal{M}_{\alpha}$. By Theorem 2.14, $\{x\}$ is $\mu$-rare or $\mu$-open. If $\{x\}$ is $\mu$-rare, then $c_{\alpha}(\{x\})=\{x\} \cup c_{\mu} i_{\mu} c_{\mu}(\{x\})=\{x\} \cup c_{\mu}(\emptyset)=$ $\{x\} \cup\left(X-\mathcal{M}_{\mu}\right)$ and so $c_{\alpha}(\{x\}) \cap \mathcal{M}_{\mu}=\{x\}$ which implies that $\{x\}$ is $\alpha$-closed in $\left(\mathcal{N}_{\alpha}, \alpha\right)$. If $\{x\}$ is $\mu$-open, then clearly, $\{x\}$ is $\alpha$-open in $\left(\mathcal{N}_{\alpha}, \alpha\right)$. Hence $(X, \mu)$ is $\alpha-T_{\frac{1}{2}}$, by Lemma 1.7. Conversely, suppose $(X, \mu)$ is $\alpha-T_{\frac{1}{2}}$ space. Suppose $x \in \mathcal{M}_{\sigma}=X$. If $x \notin \mathcal{M}_{\alpha}$, then $i_{\mu} c_{\mu}(\{x\})=i_{\mu}\left(X-\mathcal{M}_{\alpha}\right)=\emptyset$ and so $\{x\}$ is $\mu$-rare. If $x \in \mathcal{M}_{\alpha}$, then $\{x\}$ is either $\alpha$-open or $\alpha$-closed in $\left(\mathcal{M}_{\alpha}, \alpha\right)$, by Lemma 1.7. If $\{x\} \in \alpha$, then $\{x\} \in \sigma$ and so $i_{\mu}(\{x\}) \neq \emptyset$. Therefore, $\{x\} \in \mu$. If $\{x\}$ is $\alpha$-closed, then $i_{\mu} c_{\mu}(\{x\}) \subset c_{\mu} i_{\mu} c_{\mu}(\{x\}) \subset\{x\}$ and so $i_{\mu} c_{\mu}(\{x\})$ is either $\emptyset$ or $\{x\}$. Hence $\{x\}$ is either $\mu$-rare or $\mu$-open. By Theorem 2.14, $(X, \mu)$ is a $\sigma-T_{\frac{1}{2}}$ space.

Corollary 2.19. Let $(X, \mu)$ be a quasi-topological space. Then the following are equivalent.

(a) $(X, \mu)$ is $\alpha-T_{\frac{1}{2}}$.

(b) $(X, \mu)$ is $\sigma-T_{\frac{1}{2}}$.

(c) $(X, \mu)$ is $\sigma-T_{D}$.

(d) $(X, \mu)$ is $\alpha-T_{D}$.

Proof. (a) and (b) are equivalent by Theorem 2.18.

(b) and (c) are equivalent by Theorem 2.16 .

(a) implies (d) by Lemma 2.15(b) and so (c) $\Rightarrow(d)$. By Lemma 2.15(c), (d) $\Rightarrow(\mathrm{c})$ and so (c) and (d) are equivalent.

Corollary 2.20. A quasi-topological space $(X, \mu)$ is a $\alpha-T_{D}$ space if and only if $(X, \mu)$ is a $\sigma-T_{D}$ space.

A space $(X, \mu)$ is said to be $\kappa$-symmetric for $\kappa \in\{\mu, \alpha, \sigma, \pi, b, \beta\}$, if for any $x, y \in \mathcal{M}_{\kappa}, x \in c_{\kappa}(\{y\}) \cap \mathcal{M}_{\kappa}$ implies $y \in c_{\kappa}(\{x\}) \cap \mathcal{M}_{\kappa}$. The following Theorem 2.21 shows that in $\kappa$-symmetric spaces, the concepts $\kappa-T_{i}$ for $i=0, \frac{1}{2}, D, 1$ are equivalent.

Theorem 2.21. Let $(X, \mu)$ be a $\kappa$-symmetric space for $\kappa \in\{\mu, \alpha, \sigma, \pi, b, \beta\}$. Then the following are equivalent.

(a) $\mathrm{X}$ is $\kappa-T_{1}$. 
(b) $\mathrm{X}$ is $\kappa-T_{\frac{1}{2}}$.

(c) $\mathrm{X}$ is $\kappa-T_{D}$.

(d) $\mathrm{X}$ is $\kappa-T_{0}$.

Proof. It is enough to prove that $(\mathrm{d}) \Rightarrow(\mathrm{a})$. Let $x, y \in X$. Suppose, there exists $G \in \kappa$ containing $x$ but not $y$. Now $G \cap\{y\}=\emptyset$ implies that $x \notin c_{\kappa}(\{y\})$. By hypothesis, $y \notin c_{\kappa}(\{x\})$ and so there is a $H \in \kappa$ such that $y \in H$ and $x \notin H$. Therefore, $X$ is a $\kappa-T_{1}$ space.

The following Theorem 2.22 gives a characterization of $\pi-T_{1}$ quasi-topological spaces.

Theorem 2.22. Let $(X, \mu)$ be a quasi-topological space. Then $(X, \mu)$ is $\pi-T_{1}$ if and only if for every $x \in \mathcal{M}_{\mu},\{x\}$ is either $\mu$-open and $c_{\mu}(\{x\}) \cap \mathcal{M}_{\mu}=\{x\}$ or $i_{\mu}(\{x\})=\emptyset$.

Proof. Suppose that the conditions hold. Let $x \in \mathcal{M}_{\mu}$. If $i_{\mu}(\{x\})=\emptyset$, then by Lemma 1.5(c), $c_{\pi}(\{x\}) \cap \mathcal{M}_{\mu}=\left(\{x\} \cup c_{\mu} i_{\mu}(\{x\})\right) \cap \mathcal{M}_{\mu}=\left(\{x\} \cup c_{\mu}(\emptyset)\right) \cap \mathcal{M}_{\mu}=$ $\left(\{x\} \cup\left(X-\mathcal{M}_{\mu}\right)\right) \cap \mathcal{M}_{\mu}=\{x\}$. If $\{x\}$ is $\mu$-open and $c_{\mu}(\{x\}) \cap \mathcal{M}_{\mu}=\{x\}$, then $c_{\pi}(\{x\}) \cap \mathcal{M}_{\mu}=\left(\{x\} \cup c_{\mu} i_{\mu}(\{x\})\right) \cap \mathcal{M}_{\mu}=\left(\{x\} \cup c_{\mu}\{x\}\right) \cap \mathcal{M}_{\mu}=\{x\}$. Hence $(X, \mu)$ is $\pi-T_{1}$. Conversely, suppose $(X, \mu)$ is $\pi-T_{1}$. Let $x \in \mathcal{M}_{\mu}$. Then $c_{\pi}(\{x\}) \cap \mathcal{M}_{\mu}=\{x\}$ which implies that $\left(\{x\} \cup c_{\mu} i_{\mu}(\{x\})\right) \cap \mathcal{M}_{\mu}=\{x\}$ and so $\{x\} \cup\left(c_{\mu} i_{\mu}(\{x\}) \cap \mathcal{M}_{\mu}\right)=\{x\}$ which implies that $c_{\mu} i_{\mu}(\{x\}) \cap \mathcal{M}_{\mu} \subset\{x\}$. If $c_{\mu} i_{\mu}(\{x\}) \cap \mathcal{M}_{\mu}=\emptyset$, since $i_{\mu}(\{x\}) \subset$ $c_{\mu} i_{\mu}(\{x\}) \cap \mathcal{M}_{\mu}$, we have $i_{\mu}(\{x\})=\emptyset$. Suppose that $c_{\mu} i_{\mu}(\{x\}) \cap \mathcal{M}_{\mu}=\{x\}$. If $i_{\mu}(\{x\})=\emptyset$, then $c_{\mu} i_{\mu}(\{x\})=c_{\mu}(\emptyset)=X-\mathcal{M}_{\mu}$ so that $c_{\mu} i_{\mu}(\{x\}) \cap \mathcal{M}_{\mu}=\emptyset$, which is not possible. Hence $i_{\mu}(\{x\}) \neq \emptyset$ and so $i_{\mu}(\{x\})=\{x\}$ which implies that $\{x\}$ is $\mu$-open. Also, $c_{\mu}(\{x\}) \cap \mathcal{M}_{\mu}=c_{\mu} i_{\mu}(\{x\}) \cap \mathcal{M}_{\mu}=\{x\}$.

The following Theorem 2.23 shows that every quasi-topological space is $\pi-T_{\frac{1}{2}}$ which generalize the existing result that every topological space is $\pi-T_{\frac{1}{2}}[6]$.

Theorem 2.23. Every quasi-topological space $(X, \mu)$ is a $\pi-T_{\frac{1}{2}}$ space.

Proof. Let $x \in \mathcal{M}_{\mu}$. If $i_{\mu}(\{x\})=\{x\}$, then $\{x\}=i_{\mu}(\{x\}) \subset i_{\mu} c_{\mu}(\{x\})$ and so $\{x\}$ is $\pi$-open in $\left(\mathcal{M}_{\pi}, \pi\right)$. Again, if $i_{\mu}(\{x\})=\emptyset$, then $c_{\pi}(\{x\}) \cap \mathcal{M}_{\mu}=(\{x\} \cup$ $\left.c_{\mu} i_{\mu}(\{x\})\right) \cap \mathcal{M}_{\mu}=\left(\{x\} \cup c_{\mu}(\emptyset)\right) \cap \mathcal{M}_{\mu}=\{x\} \cup\left(\left(X-\mathcal{M}_{\mu}\right) \cap \mathcal{M}_{\mu}\right)=\{x\}$ so that $c_{\pi}(\{x\}) \cap \mathcal{M}_{\mu}=\{x\}$ which implies that $\{x\}$ is $\pi$-closed in $\left(\mathcal{M}_{\pi}, \pi\right)$. By Lemma 1.7, $(X, \mu)$ is a $\pi-T_{\frac{1}{2}}$ space.

The proof of the following Corollary 2.24 follows from Lemma 2.15(b). For the sake of completeness, we give the proof for Corollary 2.25.

Corollary 2.24. Every quasi-topological space $(X, \mu)$ is $\pi-T_{D}$.

Corollary 2.25. Every quasi-topological space $(X, \mu)$ is $\pi-T_{0}$. 
Proof. Let $x, y \in \mathcal{M}_{\mu}$ such that $x \neq y$. By Theorem 2.23, $\{x\}$ and $\{y\}$ are either $\pi$-open or $\pi$-closed in $\left(\mathcal{M}_{\mu}, \pi\right)$. If $\{x\}$ or $\{y\}$ is $\pi$-open, then $\{x\}$ or $\{y\}$ is the required $\pi$-open containing one but not the other. Suppose $\{x\}$ and $\{y\}$ are $\pi$-closed. Since $\{x\}$ is $\pi$-closed, $c_{\pi}(\{x\}) \cap \mathcal{M}_{\mu}=\{x\}$ and so $\mathcal{M}_{\mu}-\{x\}$ is a $\pi$-open set containing $y$ but not $x$. Hence $(X, \mu)$ is $\pi-T_{0}$.

The following Theorem 2.26 shows that every quasi-topological space $(X, \mu)$ is a $b-T_{\frac{1}{2}}$ space. Corollaries 2.27, 2.28 and 2.29 below follow from Theorem 2.26.

Theorem 2.26. Every quasi-topological space $(X, \mu)$ is a $b-T_{\frac{1}{2}}$ space.

Proof. Let $x \in X=\mathcal{M}_{b}$. Suppose $x \in \mathcal{M}_{\mu}$. If $i_{\mu}(\{x\})=\{x\}$, then $\{x\}$ is $b$-open. Again, if $i_{\mu}(\{x\})=\emptyset$ and so $c_{\mu} i_{\mu}(\{x\})=X-\mathcal{M}_{\mu}$. Then $i_{\mu} c_{\mu}(\{x\}) \cap c_{\mu} i_{\mu}(\{x\})=$ $i_{\mu} c_{\mu}(\{x\}) \cap\left(X-\mathcal{M}_{\mu}\right)=\emptyset \subset\{x\}$ and so $\{x\}$ is $b$-closed. On the other hand, if $x \in X-\mathcal{M}_{\mu}$, then $i_{\mu}(\{x\})=\emptyset$ and so $c_{\mu} i_{\mu}(\{x\})=X-\mathcal{M}_{\mu}$. Then $i_{\mu} c_{\mu}(\{x\}) \cap$ $c_{\mu} i_{\mu}(\{x\})=\emptyset \cap\left(X-\mathcal{M}_{\mu}\right)=\emptyset \subset\{x\}$ and so $\{x\}$ is $b$-closed. Hence $(X, \mu)$ is $b-T_{\frac{1}{2}}$.

Corollary 2.27. Every quasi-topological space $(X, \mu)$ is a $\beta-T_{\frac{1}{2}}$ space.

Corollary 2.28. Every quasi-topological space $(X, \mu)$ is a $b-T_{D}$ space as well as a $b-T_{0}$ space.

Corollary 2.29. Every quasi-topological space $(X, \mu)$ is a $\beta-T_{D}$ space as well as a $\beta-T_{0}$ space.

The following Theorem 2.30 gives a characterization of $\beta-T_{1}$ quasi-topological spaces, and Theorem 2.31 below shows that the separation axioms $\beta-T_{1}$ and $b-T_{1}$ are equivalent in quasi-topological spaces.

Theorem 2.30. A quasi-topological space $(X, \mu)$ is $\beta-T_{1}$ if and only if for each point $x \in X$, either $\{x\}$ is $\mu r$-open or $i_{\mu}(\{x\})=\emptyset$.

Proof. $(X, \mu)$ is $\beta-T_{1}$ if and only if $\{x\}$ is $\beta$-closed for every $x \in X$ if and only if for each $x \in X, i_{\mu} c_{\mu} i_{\mu}(\{x\}) \subset\{x\}$. Now, $i_{\mu} c_{\mu} i_{\mu}(\{x\}) \subset\{x\}$ implies that either $i_{\mu} c_{\mu} i_{\mu}(\{x\})=\{x\}$ or $i_{\mu} c_{\mu} i_{\mu}(\{x\})=\emptyset$. If $i_{\mu} c_{\mu} i_{\mu}(\{x\})=\emptyset$, then $i_{\mu}(\{x\})=\emptyset$ and so either $i_{\mu}(\{x\})=\{x\}$ or $i_{\mu}(\{x\})=\emptyset$. If $i_{\mu}(\{x\})=\{x\}$, then $i_{\mu} c_{\mu}(\{x\})=\{x\}$ and so $\{x\}$ is $\mu r$-open. Conversely, if either $i_{\mu} c_{\mu}(\{x\})=\{x\}$ or $i_{\mu}(\{x\})=\emptyset$, then $i_{\mu} c_{\mu} i_{\mu}(\{x\}) \subset\{x\}$ and so $\{x\}$ is $\beta$-closed.

Theorem 2.31. A quasi-topological space $(X, \mu)$ is $\beta-T_{1}$ if and only if it is $b-T_{1}$.

Proof. If $(X, \mu)$ is $b-T_{1}$, then for each $x \in X,\{x\}$ is $b$-closed. That is, $c_{\mu} i_{\mu}(\{x\}) \cap$ $i_{\mu} c_{\mu}\left(\{x\} \subset\{x\}\right.$. But this implies that either $c_{\mu} i_{\mu}(\{x\}) \cap i_{\mu} c_{\mu}(\{x\})=\emptyset$ or $c_{\mu} i_{\mu}(\{x\}) \cap$ $i_{\mu} c_{\mu}(\{x\})=\{x\}$. If $i_{\mu}(\{x\}) \neq \emptyset$, then $i_{\mu}(\{x\})=\{x\}$ and so $c_{\mu} i_{\mu}(\{x\}) \cap i_{\mu} c_{\mu}(\{x\})=$ 
$c_{\mu}(\{x\}) \cap i_{\mu} c_{\mu}(\{x\})=i_{\mu} c_{\mu}(\{x\})$ and so $i_{\mu} c_{\mu}(\{x\})=\{x\}$ or $i_{\mu} c_{\mu}(\{x\})=\emptyset$ which implies that $\{x\}$ either is $\mu r$-open or $i_{\mu}(\{x\})=\emptyset$. Therefore, by Theorem 2.30, $(X, \mu)$ is a $\beta-T_{1}$ space. Conversely, if for every point $x$, either $i_{\mu}(\{x\})=\emptyset$ or $i_{\mu} c_{\mu}(\{x\})=\{x\}$, then $c_{\mu} i_{\mu}(\{x\}) \cap i_{\mu} c_{\mu}(\{x\}) \subset\{x\}$ so that the space is $b-T_{1}$.

The proof of the following Corollary 2.32 follows from Theorems 2.21, 2.30 and 2.31 .

Corollary 2.32. Every quasi-topological $\pi-T_{1}$ space $(X, \mu)$ is a $b-T_{1}$ space.

Theorem 2.33. Every quasi-topological $\pi-T_{D}$ space $(X, \mu)$ is a $b-T_{D}$ space.

Proof. Let $x \in X$. If $x \in \mathcal{M}_{\mu}$, then the proof is clear. Suppose that $x \in X-\mathcal{M}_{\mu}$. Then $i_{\mu} c_{\mu}(\{x\}) \cap c_{\mu} i_{\mu}(\{x\})=i_{\mu}\left(X-\mathcal{M}_{\mu}\right) \cap c_{\mu}(\emptyset)=\emptyset \cap\left(X-\mathcal{M}_{\mu}\right)=\emptyset \subset\{x\}$ and so $\{x\}$ is $b$-closed, by Lemma $1.5(\mathrm{e})$. Hence $(X, \mu)$ is $b-T_{D}$.

Theorem 2.34. For every space $(X, \mu)$, the following hold.

(a) If $(X, \mu)$ is $\mu-T_{\frac{1}{2}}$, then $(X, \mu)$ is an $\alpha-T_{\frac{1}{2}}$ space.

(b) If $(X, \mu)$ is a quasi-topological $\mu-T_{\frac{1}{2}}$ space, then $(X, \mu)$ is $\beta-T_{\frac{1}{2}}$.

Proof. (a) Suppose that $(X, \mu)$ is a $\mu-T_{\frac{1}{2}}$ space and $x \in \mathcal{M}_{\mu}$. Then either $\{x\}$ is $\mu$-open or $c_{\mu}(\{x\}) \cap \mathcal{M}_{\mu}=\{x\}$. If $\{x\}$ is $\mu$-open, then it is $\alpha$-open. Now $c_{\alpha}(\{x\}) \cap \mathcal{M}_{\mu}=\left(c_{\mu} i_{\mu} c_{\mu}(\{x\}) \cup\{x\}\right) \cap \mathcal{M}_{\mu}=\left(c_{\mu} i_{\mu} c_{\mu}(\{x\}) \cup\left(c_{\mu}(\{x\}) \cap \mathcal{M}_{\mu}\right)\right) \cap$ $\mathcal{M}_{\mu}=c_{\mu}(\{x\}) \cap\left(c_{\mu} i_{\mu} c_{\mu}(\{x\}) \cup \mathcal{M}_{\mu}\right) \cap \mathcal{M}_{\mu}=c_{\mu}(\{x\}) \cap \mathcal{M}_{\mu}=\{x\}$. Therefore, $c_{\alpha}(\{x\}) \cap \mathcal{M}_{\mu}=\{x\}$. Thus, $(X, \mu)$ is $\alpha-T_{\frac{1}{2}}$.

(b) Let $x \in X$. If $\{x\}$ is $\mu$-open, then $\{x\}$ is $\beta$-open. If $x \in \mathcal{M}_{\mu}$, then $c_{\mu}(\{x\}) \cap$ $\mathcal{M}_{\mu}=\{x\}$. Now $c_{\beta}(\{x\}) \cap \mathcal{M}_{\mu}=\left(\{x\} \cup i_{\mu} c_{\mu} i_{\mu}(\{x\})\right) \cap \mathcal{M}_{\mu}=\left(\left(c_{\mu}(\{x\}) \cap \mathcal{M}_{\mu}\right) \cup\right.$ $\left.i_{\mu} c_{\mu} i_{\mu}(\{x\})\right) \cap \mathcal{M}_{\mu}=c_{\mu}(\{x\}) \cap i_{\mu} c_{\mu} i_{\mu}(\{x\}) \cap \mathcal{M}_{\mu}=i_{\mu} c_{\mu} i_{\mu}(\{x\}) \cap \mathcal{M}_{\mu} \subset c_{\mu}(\{x\}) \cap$ $\mathcal{M}_{\mu}=\{x\}$. But $\{x\} \subset c_{\beta}(\{x\}) \cap \mathcal{M}_{\mu}$ and so $c_{\beta}(\{x\}) \cap \mathcal{M}_{\mu}=\{x\}$. If $x \notin \mathcal{M}_{\mu}$, then $c_{\beta}(\{x\})=\{x\} \cup i_{\mu} c_{\mu} i_{\mu}(\{x\})=\{x\} \cup i_{\mu} c_{\mu}(\emptyset)=\{x\} \cup i_{\mu}\left(X-\mathcal{M}_{\mu}\right)=\{x\}$. Hence $(X, \mu)$ is $\beta-T_{\frac{1}{2}}$.

Remark 2.35. The converse of some of the theorem established above are not true for topological spaces (for example, Theorems 2.33 and 2.34 and Corollaries 2.11, 2.13, 2.32 and 2.33) and so are not true for quasi-topological spaces.

Acknowledgement. The authors sincerely thank the referee for the valuable suggestions.

\section{References}

[1] Á. Császár, Generalized topology, generalized continuity, Acta Math. Hungar., 96(2002), 351 357, DOI: 10.1023/A:1019713018007. 
[2] Á. Császár, Separation Axioms for Generalized Topologies, Acta Math. Hungar., 104(2004), 63-69.

[3] Á. Császár, Generalized open sets in generalized topologies, Acta Math. Hungar., 106(2005), 53-66, DOI: 10.1007/s10474-005-0005-5.

[4] Á. Császár, Further remarks on the formula for $\gamma$-interior, Acta Math. Hungar., 113(2006), 325-332, DOI: 10.1007/s10474-006-0109-6.

[5] A. Guldurdek and O. B. Ozbakir, On $\gamma-$ semi-open sets, Acta Math. Hungar., 109(4)(2005), 347-355, DOI: 10.1007/s10474-005-0252-5.

[6] H. Maki, J. Umehara and T. Noiri, Every topological space is pre- $T_{\frac{1}{2}}$, Mem. Fac. Sci. Kochi. Univ. Math., 17(1996), 33-42.

[7] V. Pankajam and D. Sivaraj, Some separation axioms in generalized topological spaces, Bol. Soc. Paran. Mat.(3s)., 31(1)(2013), 29-42.

[8] I. L. Reilly and M.K. Vamanamurthy, On $\alpha$-sets in topological spaces, Tamkang J. Maths., 16(1)(1985), 7-11.

[9] V. Renukadevi and D. Sivaraj, On $\delta$-sets in $\gamma$-spaces, Filomat, 22(1)(2008), 95104.

[10] V. Renukadevi and D. Sivaraj, On weak separation axioms in generalized topological spaces, Annales Univ. Sci. Budapest., to appear.

[11] P. Sivagami, Remarks on $\gamma$-interior, Acta Math. Hungar., 119(2008), 81-94, DOI: 10.1007/s10474-007-7007-4. 